University at Albany, State University of New York

Scholars Archive

$12-2003$

\title{
Underdetermination and the Problem of Identical Rivals
}

P.D. Magnus

University at Albany, State University of New York, pmagnus@albany.edu

Follow this and additional works at: https://scholarsarchive.library.albany.edu/cas_philosophy_scholar

Part of the Philosophy Commons

\section{Recommended Citation}

P. D. Magnus . "Underdetermination and the Problem of Identical Rivals." Philosophy of Science 2003; 70(5), 1256-1264. DOI: 10.1086/377405

This Article is brought to you for free and open access by the Philosophy at Scholars Archive. It has been accepted for inclusion in Philosophy Faculty Scholarship by an authorized administrator of Scholars Archive. For more information, please contact scholarsarchive@albany.edu. 


\title{
Underdetermination and the Problem of Identical Rivals
}

\author{
P. D. Magnus ${ }^{\dagger}$
}

\begin{abstract}
If two theory formulations are merely different expressions of the same theory, then any problem of choosing between them cannot be due to the underdetermination of theories by data. So one might suspect that we need to be able to tell distinct theories from mere alternate formulations before we can say anything substantive about underdetermination, that we need to solve the problem of identical rivals before addressing the problem of underdetermination. Here I consider two possible solutions: Quine proposes that we call two theories identical if they are equivalent under a reconstrual of predicates, but this would mishandle important cases. Another proposal is to defer to the particular judgements of actual scientists. Consideration of an historical episode - the alleged equivalence of wave and matrix mechanics - shows that this second proposal also fails. Nevertheless, I suggest, the original suspicion is wrong; there are ways to enquire into underdetermination without having solved the problem of identical rivals.
\end{abstract}

1. Introduction. Naturally, underdetermination can only arise between two or more rival theories. Underdetermination arguments differ as to how the rivals are filled in; some provide specific examples, some provide algorithmically generated rivals, and others invoke the promise of some as yet unappreciated rival. Regardless, they must provide some viable option besides endorsing one particular theory.

This precondition for underdetermination is simple enough to specify formally, but more difficult to apply. Take a simple example of two atomic theories. The first is the usual theory according to which 'electrons' are negatively-charged particles at the periphery of atoms and 'protons' are

$\dagger$ To contact the author, write to Department of Philosophy, Bowdoin College, 8400 College Station, Edward Pols House, 5 Bath Road, Brunswick, ME 04011-8484, pmagnus@fecundity. com.

$\ddagger$ In writing and revising this paper, I benefitted from helpful conversations with Craig Callender, Jonathan Cohen, and a number of fellow graduate students. Part of the work was done with the support of a National Science Foundation graduate fellowship. A version of this paper was presented to the Southern California Philosophy Conference in October 2001.

Philosophy of Science, 70 (December 2003) pp. 1256-1264. 0031-8248/2003/7005-0033\$10.00

Copyright 2003 by the Philosophy of Science Association. All rights reserved. 
positively-charged particles in the core of atoms. The other maintains, contrarily, that 'electrons' are positively-charged particles in the core of atoms and that 'protons' are negatively-charged particles at the periphery of atoms. Suppose further that the latter theory attributes every feature to the 'electron' that the former attributes to the 'proton' and vice versa. The latter theory includes the claim, for instance, that the 'electron' has the same mass as the 'neutron.' How do these two fare as rivals?

It is tempting to say that these are not even distinct theories. The Russian or French translations of our usual atomic theory are nevertheless the same theory as the first theory we are considering. So, too, we might insist that the second theory is just another formulation of the first- not in Russian or French, but in its own obscurantist argot. If the two were merely translations of one another, then we wouldn't have distinct theories and a fortiori we would not have rival theories. If this were so, it would make no sense to ask whether the choice between the two was underdetermined; the answer would be trivially negative. If they were really distinct, however, then the choice between them is plausibly underdetermined. So, identifying underdetermination seems to turn on telling whether two theories are merely notational variants. ${ }^{1}$

Put the worry this way: we can't reasonably say whether the choice between two rival theories is underdetermined if the two putative rivals might only be rival formulations of one theory. Without criteria of theory identity and non-identity, talk of underdetermination is premature. Identifying when putative rivals are merely alternative formulations of the same theory is not an easy thing. If we give it the somewhat paradoxical label the problem of identical rivals, then the worry is that resolving the problem of identical rivals is a necessary condition for a serious discussion of underdetermination.

2. Quine's Solution. This worry motivates Quine, who considers cases like this electron-proton inversion and suggests, just as I have, that they be treated as two formulations of one theory $(1975,319-320)$. He generalizes from these cases and proposes that "two formulations express the same theory if they are empirically equivalent and there is a reconstrual of predicates that transforms the one theory into a logical equivalent of the other" $(1975,320){ }^{2}$ A reconstrual of predicates is a mapping from the

1. Horwich (1982) considers such cases and claims on grounds of common usage that theories like the second are false. Like the present suggestion, this avoids underdetermination between alleged rivals just when we have a way to tell that alleged rivals are notational variants.

2. Sklar (1985) worries that intertranslatability criteria like Quine's prove spurious - that they amount to nothing over and above empirical equivalence - but I will presume for the sake of argument that they add something. 
predicates of one language onto the open formulae of the other, such that each $n$-place predicate is mapped onto a formula with $n$ free variables. In our example, the reconstrual is straightforward: map the predicate electron onto the sentence ' $\mathrm{x}$ is a proton' and vice versa.

Quine goes on to consider "a less trivial case," one which he attributes to Poincaré-more on Poincaré in a moment. The example involves two cosmologies: "Here we have one formulation of cosmology that represents space as infinite, and another formulation that represents space as finite but depicts all objects shrinking in proportion as they move away from the center." Quine insists that these two cosmologies are alternative formulations of the same theory just as our two 'atomic theories' were. He does not specify how this is so, but instead asserts that the reconstrual, although "less simple," "presents no serious challenge" $(1975,322)$. Quine leaves this as an exercise for the reader, but the reader may well be puzzled.

There is a serious - perhaps insurmountable - challenge which suggests that Quine's claim about these two cosmologies is simply false. The latter cosmology, we may expect, has a predicate 'Point $x$ is the center of the universe' which is satisfied by exactly one point. How are we to reconstrue this in the idiom the first cosmology, an idiom in which no point enjoys such a unique status? If there is no such reconstrual, then the two cosmologies are distinct theories even by Quine's own criterion. ${ }^{3}$

Although Quine's example misfires, we can easily provide one more suited to his purposes. Let two cosmologies be given in this way:

$\mathbf{C}_{1}$ Spacetime has some geometry $G$, and everything in spacetime follows such-and-so inertial trajectories.

$\mathbf{C}_{2}$ Spacetime has some alternate geometry $G^{*}$, and universal forces act on everything in spacetime such that all trajectories conform to $\mathrm{C}_{1}$.

Here we may have one cosmology that represents the curved spacetime of general relativity and another that represents a flat spacetime plus universal forces. It looks as if $\mathrm{C}_{1}$ and $\mathrm{C}_{2}$ are identical on Quine's criterion. We can take any claim about geometry in $\mathrm{C}_{1}$ and map it onto a claim about physical geometry in $\mathrm{C}_{2}$, and so on. Of course, this rivalry provides the basis for Reichenbach's argument that geometry is underdetermined by physical theory (1951, ch. 8; 1958). Several authors, presuming the cosmologies to be distinct, have dared to argue that one is better confirmed that the other! ${ }^{4}$ We cannot even take them seriously if the cosmologies are not rival theories. Usefulness might distinguish one

3. The fact that no observer in the second cosmology could determine which point satisfies this predicate is irrelevant. Saying the two theories are identical is stronger than saying that they practically indistinguishable.

4. For instance, Glymour (1980, ch. 9) and Grünbaum (1960). 
formulation from another, but confirmation will not. Thus, it seems that consequences of Quine's identity criterion are altogether too strong. ${ }^{5}$

Quine does not say where Poincaré offers his example, but Poincaré surely does offer cases like it. He writes, for instance, of

two universes which are the image one of the other. With each object $P$ in the universe $A$, there corresponds, in the universe $B$, an object $P^{1}$ which is its image. The co-ordinates of this image $P^{1}$ are determinate functions of the object $P$; moreover, these functions may be of any kind whatever. ... ([1908] 1952, 98)

Poincare maintains not that the claim 'We are in universe $A$ ' and the claim 'We are in universe $B$ ' are the same claim, formulated one in the language of $A$ and the other in the language of $B$; rather, he says that "these two universes will be indistinguishable" ([1908] 1952, 98). He sees this as grounds for refusing to make any claims about absolute space whatsoever. Thus, where Poincare insists that the choice of a theory about absolute geometry is underdetermined, Quine insists that it cannot be underdetermined because all the empirically adequate ones would - on final analysis - prove to be merely formulations of one theory.

3. The Naturalist Rejoinder. Quine's criterion would allow scientists to dispose of the electron-proton inversion case, but they are unlikely to take it seriously without Quine's help. Since scientists can dismiss that case without a formal justification, we might look to scientists and to the scientific community to determine which formulations represent distinct theories. This follows the advice of Larry Laudan and Jarrett Leplin, who counsel a policy of "deference to scientific judgment as to what constitutes a theory" $6(1993,13)$.

It is one thing to look at scientific practice and attempt to abstract from it principles by which to guide our enquiry. We might use insights gleaned from the bulk of science to shed light on some particular part of it. The proposal here is another thing entirely: that we should take our cue from particular scientific judgments. If we defer to science on each particular, we would not be able to dissent from any scientific judgement. We could adduce no normative principle besides 'Follow the scientific community in all things' - a principle trivially followed by the scientific community even

5. Similar conclusions are drawn by Mühlhölzer, who concludes that "Quine's criterion . . . blurs important distinctions...." $(1994,123)$.

6. Laudan and Leplin make this suggestion as a way to determine which rival theories are serious rivals, so it may not be fair to attribute its consequences to them when it is applied to theory identity. Nevertheless, looking to the scientific community to resolve disputes about theory identity is a plausible naturalist move. 
where it stumbles or goes astray. If scepticism is the Scylla of epistemology, then quietism is its Charybdis. 'Believe what you believe' is of as little practical value to a deliberating agent as 'Believe nothing.'

The suggestion may be seen as something more than quietism insofar as it recommends for philosophers to defer to scientists. Advice to defer to experts may be of use to deliberating agents who are not themselves experts. This might be a blow to the egos of philosophers but perhaps it would be all well and good, if only scientists had expertise on the identity of theories beyond the ken of philosophers. However, scientists don't centrally concern themselves with criteria of identity or meaning. They employ and criticize particular theories, but they do not by and large pay attention to theory as such. Moreover, there have been theories in the history of science which at one time were considered rivals but which came to be seen as alternate formulations of a single theory. The lesson of such episodes is that deference to scientific judgment on these matters might lead us astray.

4. The Case of Matrix and Wave Mechanics. This point is best pressed home by considering a specific example. In 1926, two formulations of quantum mechanics were on offer: matrix mechanics which had been introduced by Werner Heisenberg and others the year before and wave mechanics formulated by Erwin Schrödinger. Schrödinger and Carl Eckart independently published results which claimed to show that matrix mechanics and wave mechanics were equivalent. Thus, matrix and wave mechanics were at most rival formulations of one common theory. It is usual to say both that the two were equivalent and that the papers by Schrödinger and Eckart provided valid reason for thinking so. A typical physics textbook claims that Schrödinger "showed that the matrix and wave mechanics formulations give identical results and differ only in their mathematical form" (Thornton and Rex 1993, 208). Philosophers make similar claims, saying, for example, "The early formulations of the theory, by Heisenberg and Schrödinger, were, respectively, in terms of sequences and of functions; subsequently Schrödinger established that . . . the two formulations were equivalent." So, the two are treated by almost everyone as being merely "formulations of the [singular] theory" (Hughes 1989, 45). Such claims are so widespread as to count as common knowledge. ${ }^{7}$ The connection with underdetermination has not gone unnoticed: Philip Kitcher uses the notion that the two are merely rival formulations to deny that the choice between them was underdetermined (2001a, 195; 2001b,

7. Muller, who dubs the view the Equivalence Myth, provides a no doubt incomplete list of almost fifty sources that promulgate it $(1997,37)$. 
35). ${ }^{8}$ Yet, as Norwood Russell Hanson remarked, "the unguarded statement that Wave and Matrix Mechanics are equivalent physical theories is so unsound, historically and even conceptually, that a re-examination of the issue might still be tolerable" $(1963,113)$.

F.A. Muller employs the structural view of theories in such a reexamination and, like Hanson, concludes that Schrödinger's 1926 paper did not show what it is so often taken to show. ${ }^{9}$ Even the usual account concedes that matrix and wave mechanics were associated with different ontologies - the former quite deliberately involved no commitment to unobserved states of particles, whereas the latter treated particles as quivering puddings of mass and charge. ${ }^{10}$ This difference in ontology is not merely a matter of labels. Because waves are distributed in space, wave mechanics has the resources to express spatial relations. Apply this to a concrete case and consider a charged particle detector that occupies some specified region of space and an electron that turns up in it. Since waves disperse over time, wave mechanics predicts that the detector should not detect the entire charge of the electron. Only part of the electron wave will be in the detector, and only that part would be detected. Since there is no straightforward way to represent spatial coordinates in matrix mechanics, contrawise, matrix mechanics does not yield this prediction. This prediction might have been tested, allowing the opportunity to distinguish empirically between wave and matrix mechanics (Muller 1997, 227). Rather than being mere window dressing, the differing ontologies reflect differences in expressive power and empirical upshot. With the further notion of wave collapse - the supposition that the wave becomes localized when it is observed - wave mechanics would not yield the critical prediction. Be that as it may, the notion of wave collapse had not been introduced at the time of Schrödinger's alleged equivalence proof. ${ }^{11}$

This historical episode holds an important telling point against the naturalist criterion for theory identity. Scientists can believe that two theories are merely rival formulations of one theory even when they have insufficient reason for doing so. This may be self-fulfilling; if further development of each rival is directed toward making the supposed identity more explicit, then successors of the two rivals may be formulations of

8. This connection is also made by Wilson $(1980,217)$ and Sklar (1985).

9. Muller cites Hanson as someone who "denies the equivalence, but unfortunately for all the wrong reasons" (1997, $37 \mathrm{fn}$. 4). This is probably unfair, but I make no attempt here to resuscitate Hanson's reasoning.

10. In Muller's phrase, “tiny jelly-like lumps of vibrating charged matter” (1997, 229).

11. One may say that Schrödinger's theory of waves qua matter waves was simply falsified by subsequent developments. 
some one theory even if the initial rivals were not. Surely, it is also possible for two rivals to be rival formulations of one theory even though scientists do not know that to be the case.

5. Taking Stock. Muller's work using the structural conception of theories provides an example of how specific questions of theory equivalence may be resolved, so one might think that it shows how the problem of identical rivals may be solved in general. This structural conception (called variously the semantic or model-theoretic conception) requires specifying the theory as a class of set-theoretical structures (or models). Critics have charged that this formal requirement elides important features of scientific theories (Cartwright 1983, 159-161), and even advocates of the semantic conception argue for treating theories as 'models' in an informal, nonmathematical sense. Giere, for instance, argues for the "model-theoretic view" and allows models that are "prototypes or exemplars" (1994, 283, fn. 3). Where models are not or cannot be specified set-theoretically, Muller's approach can find no purchase. Thus relying on the structural conception here would not provide a general solution and would replace one dispute with another.

Without attempting to decide between the structural view and its many rivals, ${ }^{12}$ I remind the reader that theories are products of human craftsmanship. Not only do theories have histories, but theory itself has a history. Whatever it is now, it came to be that way and may come to be different. Philosophers can accompany an account of theory with a call to represent theories in that way, ${ }^{13}$ but theory as scientists meet it is red in tooth and claw. Closer examination of episodes like the dissolving rivalry between wave and matrix mechanics might allow us to adduce principles of theory identity and non-identity - principles informed by and applicable in practice. Let me simply observe that questions of theory identity are unresolved and that their resolution (if it is possible at all) would demand considerable further work.

I concluded Section 1 with the worry that solving the problem of identical rivals was a precondition for talking seriously about underdetermination. Absent such a solution, should underdetermination be taboo? I think not. Any two theory formulations which a scientist takes to be distinct can be considered as rivals for the purpose of asking if the choice between them is underdetermined. This enquiry might be fruitful in one of two ways. First, if the choice between rivals can be shown not to be

12. Formal work has also been done on the identity conditions of theories within state-space semantics (Churchland 1998), but one is hard-pressed to see how the theories considered above could be reconstrued as patterns of activations in neural networks.

13. As Suppes (1968) does. 
underdetermined, then the theories must be distinct. Even if we don't know necessary and sufficient conditions for theory identity, we do know some sufficient conditions for non-identity - empirical inequivalence, for one. Second, if the alleged underdetermination can be shown to have no serious consequences, then nothing turns on whether the rivals are actually distinct. Even if the electron-proton inversion case involved distinct theories in some sense, it would be hard to get excited about the underdetermination between them.

It is possible, of course, that there will be some cases of putative underdetermination with considerable consequences. We might then be pushed to consider whether the apparent underdetermination obtains between distinct theories - pushed, that is, to consider the problem of identical rivals. Whether that will happen is not something we can know a priori, however, so the worry should not paralyze us.

I do not deny that a criterion of theory identity would be a nice thing to have. Problems of theory individuation, of which the problem of identical rivals is a special case, are interesting in their own right. Resolving them, however, can only come as the result of a careful examination of the history of science - an examination which must be left for some other time. I draw the modest conclusion that this open question need not turn us back from considering underdetermination.

\section{REFERENCES}

Cartwright, Nancy (1983), How the Laws of Physics Lie. Oxford: Oxford University Press. Churchland, Paul (1998), "Conceptual Similarity Across Sensory and Neural Diversity", in On the Contrary. Cambridge, MA: MIT Press, 81-112.

Giere, Ronald N. (1994), "The Cognitive Structure of Scientific Theories", Philosophy of Science 61:276-296.

Glymour, Clark (1980), Theory and Evidence. Princeton, NJ: Princeton University Press.

Grünbaum, Adolf (1960), "The Duhemian Argument", Philosophy of Science 27:75-87.

Hanson, Norwood Russell (1963), The Concept of the Positron. Cambridge: Cambridge University Press.

Horwich, Paul (1982), "How to Choose between Empirically Indistinguishable Theories", The Journal of Philosophy 79:61-77.

Hughes, R. I. G. (1989), The Structure and Interpretation of Quantum Mechanics. Cambridge, MA: Harvard University Press.

Kitcher, Philip (2001a), "Real Realism: The Galilean Strategy", The Philosophical Review 110:151-197. (2001b), Science, Truth, and Democracy. Oxford: Oxford University Press.

Laudan, Larry, and Jarrett Leplin (1993), "Determination Undeterred: Reply to Kukla", Analysis 53:8-16.

Mühlhölzer, Felix (1994), "Scientific Explanation and Equivalent Descriptions", in W. Salmon and G. Walters (eds.), Logic, Language, and the Strucuture of Scientific Theories. Proceedings of the Carnap-Reichenbach Centennial, University of Konstanz, 21-24 May 1991, 119-138. Pittsburgh: University of Pittsburgh Press.

Muller, F. A. (1997), "The Equivalence Myth of Quantum Mechanics", Studies in the History and Philosophy of Modern Physics 28:35-61, 219-247.

Poincaré, Henri ([1908] 1952), Science and Method. Translated by Francis Maitland. New York: Dover. 
Quine, Willard Van Orman (1975), "Empirically Equivalent Systems of the World", Erkenntnis 9:313-328.

Reichenbach, Hans (1951), The Rise of Scientific Philosophy. Berkeley: University of California Press.

(1958), The Philosophy of Space \& Time. Translated by Maria Reichenbach and John Freund. New York: Dover.

Sklar, Lawrence (1985), "Saving the Noumena", in Philosophy \& Spacetime Physics, 4972. Berkeley: University of California Press.

Suppes, Patrick (1968), "The Desirability of Formalization in Science", The Journal of Philosophy 65:651-664.

Thornton, Stephen T., and Andrew Rex (1993), Modern Physics for Scientists and Engineers. Fort Worth, TX: Saunders College Publishing.

Wilson, Mark (1980), "The Observational Uniqueness of Some Theories", The Journal of Philosophy 77:208-233. 